\section{e0142 THE EFFECTS OF STROMAL VASCULAR FRACTION FROM ADIPOSE TISSUE ON RAT ACUTE MYOCARDIAL INFARCTION}

doi:10.1136/hrt.2010.208967.142

Zhuang Wei, Xie Liangdi, Xu Changsheng. The First Affliated Hospital of Fujian Medical University, Fujian Hypertension Institute, Fuzhou, China

Objective To observe the effects of transplantation of cultured stromal vascular fraction (SVF) on infarcted myocardial tissue, cardiac function and the underling mechanism.

Design and methods Rats were randomly divided into three groups: sham-operated group, AMI control group and SVF transplantation group. SVF labelled with GFP after 3 days or PBS was injected into infarcted tissue. 2 weeks after transplantation, cardiac function was evaluated with homodynamic measurement. Fluorescence microscope was used to identify the survival of SVF cells in heart. CD31 were determined by immunohistochemitry and infarction value fraction of left ventricle was calculated by H-E straining.

Results 1 . Homodynamic measurements showed that -dp/dtmax was significantly decreased and LVDP was increased in infarction control group 2 weeks after SVF transplantation compared with infarction control group. While the two groups compared with sham-operation group, LVDP and -dp/dtmax were worse. 2. GFP labelled SVF were found in myocardial infracted zone. 3. Compared with those in the infarction control group, the construction of myocardial tissues in SVF transplantation group recovered better. 4. Endothelial marker CD31-positive cells per eyeshot were accounted. It is higher in the SVF transplantation group than that in infarction control group.

Conclusions 1 . The lentivirus vector with GFP may be used as trace marker of the transplantation cells. 2. SVF transplantation may survive in infracted heart and reduce myocardial infarction size. 3. SVF may ameliorate cardiac function and promote vascular proliferation in infraction area in AMI rat.

\section{e0143 IN VIVO STUDY OF ADENVIRUS MEDIATED TRANSGENIC HIF-1 $\alpha$ AND ITS PROTECTIVE EFFECTS ON CARDIAC FUNCTION IN EXPERIMENTAL MYOCARDIAL INFARCTION}

doi:10.1136/hrt.2010.208967.143

Li Dongye, Yan Yan, Liu Yina, Liu Chuang, Zhu Hong, Pan Defeng, Zhang Zhuoqi, Xia Yong. The Affiliated Hospital of Xuzhou Medical College, Xuzhou, Jiangsu, China

Hypoxia inducible factor- $1 \alpha$ (HIF-1 $\alpha$ ) plays important roles in the regulation of transcriptional responses to hypoxia. In this study, the expression of HIF-1 $\alpha$ gene and its downstream gene vascular endothelial growth factor (VEGF) in ischaemic myocardium and the effects of adenovirus mediated transgenic HIF-1 $\alpha$ on cardiac function and myocadiocyte apoptosis after acute myocardial infarction (AMI) were investigated. Rabbits performed experimental AMI were divided into four groups randomly and Ad-HIF-1 $\alpha$, Ad-Blank and Rz-HIF- $1 \alpha$ were transfected respectively. Sham group was regarded as control. The ultramicrostructure of ischaemic myocardium was observed by electron microscope and light microscope. The mRNA expression of HIF- $1 \alpha$ and VEGF was detected by RTPCR at different time point. The protein expression of HIF- $1 \alpha$, VEGF and CD31was detected by the means of immunohistochemistry and western-blot. The cardiac function of rabbits in each time point were detected by Maclab/8s. Cardiomyocyte apoptosis was detected with TUNEL method at different time point. It was found that the mRNA and protein expression of HIF-1 $\alpha$ and VEGF increased at $1 \mathrm{~d}$ post-infarction, with the peak at $7 \mathrm{~d}$ and decreased gradually at 14 and $28 \mathrm{~d}$. At $56 \mathrm{~d}$, HIF-1 $\alpha$ mRNA and protein were undetectable, but VEGF mRNA and protein still expressed. The cardiac functional parameter was the highest in Sham group, and the lowest in Rz-HIF- $1 \alpha$ group. It was higher in Ad-HIF- $1 \alpha$ than in Ad-Blank group. The number of apoptotic cells was the most in RzHIF-1 $\alpha$ group, and it was less in Ad-HIF-1 $\alpha$ than in Ad-Blank group. The number of apoptotic cells decreased with extension of time. There was little apoptotic cells in Sham group. From all above, it was concluded that Ad-HIF- $1 \alpha$ could be tranfected and activated effectively. Rz-HIF- $1 \alpha$ could suppress the expression of HIF- $1 \alpha$ and VEGF. The cardiac function could be improved by Ad-HIF- $1 \alpha$ and deteriorated by Rz-HIF-1 $\alpha$. Cardiomyocyte apoptosis could be inhibited by Ad-HIF-1 $\alpha$ and increased by Rz-HIF-1 $\alpha$.

\section{Q0144 THE EFFICACY OF TRANSPLANTATION OF MESENCHYMAL STEM CELLS WITH GELATIN MICROSPHERES CONTAINING VASCULAR ENDOTHELIAL GROWTH FACTOR IN ISCHAEMIC REGIONS}

doi:10.1136/hrt.2010.208967.144

Liu Qiong, Zhao Shihua, Jiang Shiliang, Lu Minjie, Ling Jian, Zhan Yan, Yan Chaowu, Cheng Huaibing, Ma Ning, Li Shiguo, Yin Gang. Department of Radiology, Cardiovascular Institute and Fuwai Hospital, Chinese Academy of Medical Sciences, Peking Union Medical College, Beijing, China

Objective The effects of cell transplantation on the ischaemic failing heart have already been documented. However, the area in and around infarct regions is not a good environment for cells to survive in because they are exposed to poor conditions in which certain requirements cannot be adequately supplied. We therefore designed a study to investigate the efficacy of transplantation of mesenchymal stem cells with gelatin microspheres containing vascular endothelial growth factor in ischaemic regions.

Methods 12 Chinese mini swines with infarction were randomised into two groups: six swines received autogenetic mesenchymal stem cells (MSCs) to the pefi-infarction area of left ventricular wall (control group), six received mesenchymal stem cell transplantation and gelatin hydrogel microspheres incoporating vascular endothelial growth factor (VEGF-MSCs group). 3 weeks later, left ventricular function was assessed by means of (MRI. The contrast of the MSCs hypointense lesion was determined using the difference in signal intensity between the hypointense and normal myocardium divided by signal intensity of the normal region.

Results The mean diameter of the microspheres is $(104 \pm 22.6) \mu \mathrm{m}$. At $24 \mathrm{~h}$, injection sites of MSCs were identified by MRI as large intramyocardial signal voids that persisted at 3 weeks. Between two groups, there were no significant difference in the contrast of the lesions and in the size of the lesions at $24 \mathrm{~h}$. At 3 weeks after injection, the size of the lesions diminished $(p<0.0001)$ and the contrast of the lesion decreased $(p<0.0001)$. Histology (at 3 weeks) revealed the iron inclusion from Prussian Blue staining matches DAPI fluorescent dyes on adjacent histological sections at $\times 40$ magnification at 3 weeks after MSCs injection, indicating partial ferumoxide particles is still contained within original MSCs. In the MSCs-VEGF microsphere group, the capillary density of the injection site was significantly more than that in MSCs group (42 \pm 13.9 / HPF vs $29 \pm 15.4 / \mathrm{HPF}, \mathrm{p}<0.0001)$. There were much more dense fluorescently labled MSCs per high power fields in injection sites of MSCs-VEGF microsphere group than that in injection sites of MSCs group (354 $\pm 83 / \mathrm{HPF}$ vs $278 \pm 97 / \mathrm{HPF}, \mathrm{p}<0.0001)$. Moreover, the apoptosis rate of MSCs of MSCs-VEGF microsphere group is more than that of MSCs group $(6 \pm 4.1) \%$ vs $(11 \pm 4.8) \%, \mathrm{p}<0.0001)$.

Conclusions VEGF incorporated microspheres benefits to the survival of MSCs transplanted to ischaemic myocardium. Microenvironment if one of the most important factors that influence the survival of transplanted MSCs. 